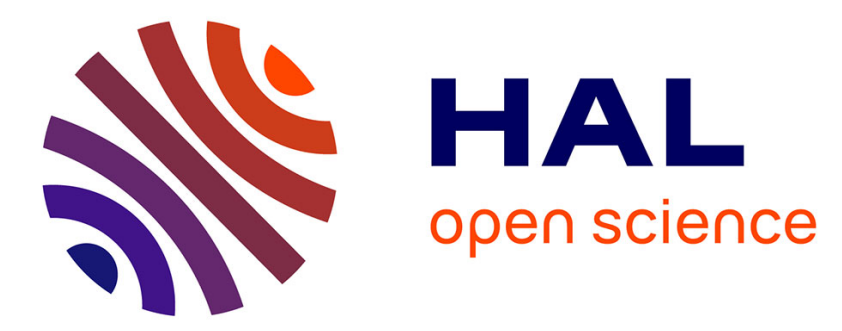

\title{
Uncoupled and coupled approaches to predict macrocrack initiation in fiber reinforced ceramic matrix composites
}

\author{
François Hild, Per-Lennart Larsson, Frederick A Leckie
}

\section{- To cite this version:}

François Hild, Per-Lennart Larsson, Frederick A Leckie. Uncoupled and coupled approaches to predict macrocrack initiation in fiber reinforced ceramic matrix composites. Composite Structures, 1994, 29 (4), pp.365-377. 10.1016/0263-8223(94)90104-X . hal-02342210

\author{
HAL Id: hal-02342210 \\ https://hal.science/hal-02342210
}

Submitted on 31 Oct 2019

HAL is a multi-disciplinary open access archive for the deposit and dissemination of scientific research documents, whether they are published or not. The documents may come from teaching and research institutions in France or abroad, or from public or private research centers.
L'archive ouverte pluridisciplinaire HAL, est destinée au dépôt et à la diffusion de documents scientifiques de niveau recherche, publiés ou non, émanant des établissements d'enseignement et de recherche français ou étrangers, des laboratoires publics ou privés. 


\title{
Uncoupled and coupled approaches to predict macrocrack initiation in fiber reinforced ceramic matrix composites
}

\author{
François Hild, ${ }^{*}$ Per-Lennart Larsson, $\ddagger \&$ Frederick A. Leckie \\ Department of Mechanical and Environmental Engineering, University of California, Santa Barabara, CA 93106, USA
}

\begin{abstract}
Localized fiber pull-out is one of the fracture features of fiber reinforced ceramic matrix composites. The onset of this mechanism is predicted by using Continuum Damage Mechanics, and corresponds to a localization of the deformations. After deriving two damage models from a uni-axial bundle approach, and criteria at localization, different axisymmetric configurations are analyzed through two different approaches to predict macrocrack initiation.
\end{abstract}

\section{INTRODUCTION}

To achieve the new goals of high performance structures, Ceramic Matrix Composites (CMCs) become a suitable candidate, especially when the elements are subjected to high mechanical and thermal load histories.' Indeed, their low density combined with high strength and good performances at high temperature are appealing features. For example, in the design of the new generation of jet engines, CMCs will be used in the combuster, the turbine disks and the nozzle section.'

In this paper we will focus our attention on rotating parts, i.e. an element in the turbine stages where angular rotation induces mechanical loading. To simplify the analysis, we will consider axisymmetric structures. The structures are assumed to be reinforced by fibers in the circumferential direction. The main goal of this paper is to predict the initiation of a macrocrack in the structure, which often constitutes the early stages of the final failure by fracture of the structure. Starting from a material which is assumed free from any initial macro defect, the initiation can be predicted using Continuum Damage Mechanics (CDM). In this paper we will neglect the matrix cracking process. This degradation takes place at an early stage of

* Laboratoire de Mécanique et Technologie, E.N.S. de Cachan/C.N.R.S./Université Paris 6, 61 avenue du Président Wilson, F-94235 Cachan Cedex, France.

$\ddagger$ Department of Solid Mechanics, Royal Institute of Technology, S-100 44 Stockholm, Sweden. loading and often does not lead to final failure of a structure. Matrix cracks gradually develop as the load level increases. They usually saturate because of the shear effects induced by the interface between fibers and matrix. ${ }^{2}$ The results presented herein are valid when the steady matrix stress $^{3}$ is less than the ultimate strength of a fiber reinforced composite. ${ }^{4}$ When that hypothesis is satisfied, the key mechanism leading to a final failure is fiber breakage. The fiber breakage mechanism is accompanied by distributed fiber pull-out; a broken fiber pulls out of the matrix and involves shear stresses along the interface to recover its original load level. This mechanism distinguishes the behavior of fiber reinforced CMCs from classical fiber-bundle behavior. ${ }^{5}$ From a design point of view it is interesting to evaluate their differences, especially in terms of load levels.

The degradation mechanism will be described by an internal variable called damage. In the framework of CDM, the initiation of a macrocrack corresponds to a localization of the deformations, which corresponds to the onset of a surface across which the velocity gradient is discontinuous. Physically, it corresponds to localized pull-out, whereby one macrocrack develops and pull-out continues to evolve in the vicinity of that macrocrack only. This phenomenon leads to a different behavior, compared to the homogeneous solution for which damage is still evolving in a diffusive manner. Under a small-deformations 
assumption, localization is mainly driven by the damage mechanism that causes strain softening.

In Section 2, the main results concerning loss of uniqueness and localization will be recalled. In particular a property giving a necessary and sufficient condition of loss of uniqueness and localization will be proven and will be applied in Section 3. Section 3 deals with two constitutive laws modeling fiber breakage. The condition of localization and loss of uniqueness will be studied, and general criteria may be derived using the property given in Section 2. Section 4 presents two strategies to predict the initiation of a macrocrack. The first one, which is referred to as fully coupled approach, consists of calculating the stresses and strains evolution in elasticity coupled with damage; therefore the damage evolution is fully coupled with the evolution of the stresses and the strains. The second approach, which is referred to as decoupled approach and which is an easier calculation more amenable to design, consists of an elastic computation and the use of the failure criterion derived from the localization analysis in Section 3. The differences between the two approaches are analyzed. The results, in terms of loads at localization, are also compared to the corresponding cumulative failure probability when the structure is assumed to be made of a brittle material.

\section{LOCALIZATION AND LOSS OF UNIQUENESS}

\subsection{General theory}

The failure at a meso-level, which corresponds to the initiation of a macrocrack, is defined as the bifurcation of the rate problem in certain modes, viz. the appearance of a surface across which the velocity gradient is discontinuous. ${ }^{7}$ This phenomenon is referred to as localization, and corresponds to the failure of the ellipticity condition. ${ }^{8}$ The condition of localization can also be compared to the loss of uniqueness of the rate problem.

Stationary waves were studied by Hadamard ${ }^{9}$ in elasticity and by Hill ${ }^{10}$ and Mandel ${ }^{11}$ in elastoplasticity. Rice ${ }^{12}$ related the localization of plastic shear bands to jumps of the velocity gradient. Borré and Maier, ${ }^{13}$ who extended the results given by Rice ${ }^{12}$ and Rice and Rudnicki, ${ }^{14,15}$ have given necessary and sufficient conditions for the onset of modes inside the body.
Under the small strains assumption and in elasticity coupled with damage, the behavior of a material is assumed to be described by the following piece-wise linear rate constitutive law

$$
\dot{\sigma}=\left\{\begin{array}{l}
\mathbf{E}: \dot{\varepsilon} \text { if } \dot{D}=0 \\
\mathbf{H}: \dot{\varepsilon} \text { if } \dot{D} \neq 0
\end{array}\right.
$$

where $\dot{\sigma}$ and $\dot{\varepsilon}$ denote the stress and strain rates, respectively, $\mathbf{E}$ and $\mathbf{H}$ are fourth-rank tensors, $\mathbf{E}$ is assumed to be positive, definite, and $D$ is either a single damage variable or a set of damage variables.

Localization occurs inside the body, if and only if $^{13,14}$

$$
\operatorname{Det}(\mathbf{n} \cdot \mathbf{H} \cdot \mathbf{n})=0
$$

for any vector $\mathbf{n} \neq 0$ and at any point inside a structure $\Omega$.

This criterion corresponds to the failure of the ellipticity condition of the rate equilibrium equation; it also can be used as an indicator of the local failure of the material, at a meso-scale.?

Furthermore, any loss of uniqueness, considered as bifurcation of the rate boundary value problem, is excluded provided the operator

$$
\mathbf{H}_{\mathrm{S}}=\frac{1}{2}\left(\mathbf{H}+\mathbf{H}^{\mathrm{r}}\right)
$$

is strictly positive-definite everywhere within the structure (where ${ }^{\mathrm{T}}$ denotes the Euclidean transposition). This condition is equivalent to the condition of hardening

$$
\dot{\sigma}: \dot{\varepsilon}>0 \text {. }
$$

Equations (2) and (3) show that the quantity that defines loss of uniqueness and localization is the linear tangent modulus $\mathbf{H}$. In the following, we analyze loss of uniqueness and loss of ellipticity (i.e. localization) for plane states when

$$
\left\{\begin{array}{l}
\varepsilon_{11}=\alpha \varepsilon_{22} \text { with } \alpha \in \mathbb{R} \\
\varepsilon_{12}=0 .
\end{array}\right.
$$

The parameter $\alpha$ is referred to as strain ratio. These particular states only are considered since the computations will deal with shear-free states. These states lead to a tangent modulus that takes the following form

$$
\mathbf{H}=\left\{\begin{array}{lll}
H_{1111} & H_{1122} & 0 \\
H_{2211} & H_{2222} & 0 \\
0 & 0 & H_{1212}
\end{array}\right\}
$$


where $H_{i j k l}=\partial \sigma_{i j} / \partial \varepsilon_{k l}$ are the components of the tangent modulus $\boldsymbol{H}$ defined in eqn (1). For problems under hypothesis (5), the non-vanishing components of vector $\mathbf{n}$ are $n_{1}$ and $n_{2}$, and the matrix $\boldsymbol{A}=\mathbf{n} \cdot \boldsymbol{H} \cdot \mathbf{n}$ reduces to ${ }^{16}$

$A=\left\{\begin{array}{ll}n_{1}^{2} H_{1111}+n_{2}^{2} H_{1212} & n_{1} n_{2}\left(H_{1212}+H_{1122}\right) \\ n_{1} n_{2}\left(H_{1212}+H_{2211}\right) & n_{1}^{2} H_{1212}+n_{2}^{2} H_{2222}\end{array}\right\}$.

We rewrite $\left(n_{1}, n_{2}\right)=\langle\cos \theta, \sin \theta)$, let $X$ be equal to $\tan ^{2} \theta$, and let $Y$ be equal to $1 / X$. The localization condition (eqn (2)) can be written in terms of $X$ or $Y$

$$
\begin{aligned}
& a X^{2}+b X+c=0, a \neq 0 \\
& c Y^{2}+b Y+a=0, c \neq 0
\end{aligned}
$$

with

$$
\begin{aligned}
a= & H_{1212} H_{2222} \\
b= & H_{1111} H_{2222}-H_{1122} H_{2211}-H_{1122} H_{1212} \\
& -H_{2211} H_{1212} \\
c= & H_{1212} H_{1111} .
\end{aligned}
$$

If real positive roots are found, then the localization direction is perpendicular to the vector $\left(n_{1}\right.$, $\left.n_{2}, 0\right)=(\cos \theta, \sin \theta, 0)$, characterized by the angle $\theta$ (Fig. 1). The values of $H_{1111}, H_{2222}, H_{1122}, H_{2211}$ and $H_{1212}$ are model-dependent.

\subsection{Necessary and sufficient condition at localization}

In this section, general results concerning localization are analyzed. A necessary and sufficient condition at localization and loss of uniqueness will be derived under the following conditions. Let us assume that $H_{1122}=H_{2211}=v H_{2222}(v<\sqrt{2})$, that $H_{1111}$ and $H_{1212}$ are positive numbers and that $H_{2222}$ can become negative $\left(H_{i j k l}\right.$ are the components of the tangent operator $\boldsymbol{H}$ ). This condition will be satisfied in the models used later. It can be seen from eqns (3) and (6) that there is no loss of uniqueness, provided

$$
\lambda>0
$$

where

$$
\begin{aligned}
2 \lambda= & H_{1111}-H_{2222} \\
& +\sqrt{\left(H_{1111}-H_{2222}\right)^{2}+2 v^{2}\left(H_{2222}\right)^{2}} .
\end{aligned}
$$

Furthermore, it is noticed that when $H_{2222}$ is equal to zero, $\lambda$ is equal to zero (loss of unique-

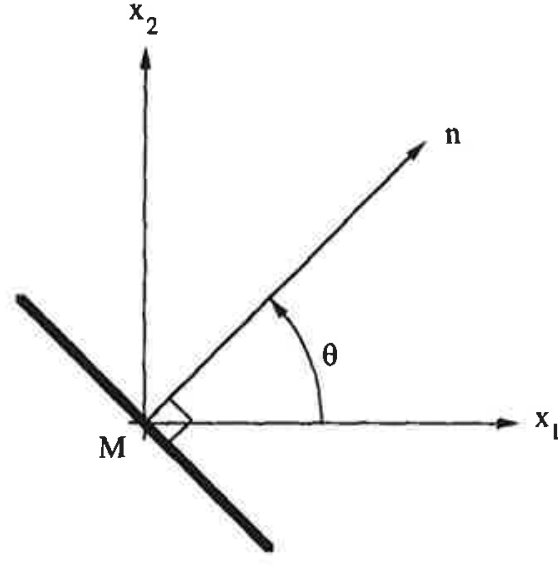

Fig. 1. Localization mode.

ness) and the coefficients $a, b$, and $c$ of eqns (9) reduce to

$$
a=0, \quad b=0, \quad c=H_{1111} H_{1212}>0 .
$$

Localization occurs since it is possible to find $Y$ satisfying eqn (8.2): $Y=0$, and the localization direction is given by $\theta=\pi / 2$. Therefore a sufficient condition of loss of uniqueness and localization is that $H_{2222}$ should be equal to zero.

Since localization corresponds to a particular mode of all of the solutions after loss of uniqueness, it suffices to show that $H_{2222}$ equal to zero is a sufficient condition for loss of uniqueness. Using the triangular inequality, we have

$$
\frac{2-v \sqrt{2}}{2} H_{2222} \leq \lambda \text {. }
$$

Loss of uniqueness corresponds to $\lambda$ equal to zero, and therefore $H_{2222} \leq 0$. Since it has been shown that $H_{2222}$ equal to zero is sufficient to define localization and loss of uniqueness, we have the following property: a necessary and sufficient condition to have localization and loss of uniqueness is $H_{2222}$ equal to zero. The angle at localization is equal to $\pi / 2$.

Conversely, under the conditions $H_{1122}=H_{2211}$ $=v H_{1111}(v<\sqrt{2}), H_{2222}$ and $H_{1212}$ are positive numbers and $H_{1111}$ can become negative, a necessary and sufficient condition to have localization and loss of uniqueness is $\mathrm{H}_{111}$ equal to zero. The angle at localization is equal to 0 . The proof is the same as previously when studying eqn (8.1).

\section{CONSTITUTIVE LAWS}

This section is concerned with the development of two constitutive laws in the case of CMCs rein- 
forced in one direction. Since these models will be used to study spinning structures, we choose a cylindrical coordinate system $(r, \varphi)$ as shown in Fig. 2(a). We assume a plane stress hypothesis. This hypothesis is consistent with the results derived in Section 2: eqn (6) is still valid. The fibers are assumed to be in the hoop direction, and the two analyzed geometries are a disk of outer radius $a$, and a ring of outer radius $a$ and inner radius $b(a=2 b)$ shown in Figs 2(b) and 2(c). We let $\omega$ denote the angular rotation speed. Since the structure, the boundary conditions and the loading are axisymmetric, the problem is axisymmetric, and the only non-zero stresses are related to the corresponding strains by ${ }^{17}$

$$
\begin{aligned}
\sigma_{r r}= & \frac{E_{\varphi}}{k\left\{1-v_{r \varphi}^{2}\left(1-D_{\varphi}\right) k\right\}} \\
& \times\left\{\varepsilon_{r r}+v_{r \varphi}\left(1-D_{\varphi}\right) k \varepsilon_{\varphi \varphi}\right\} \\
\sigma_{\varphi \varphi}= & \frac{E_{\varphi}\left(1-D_{\varphi}\right)}{1-v_{r \varphi}^{2}\left(1-D_{\varphi}\right) k}\left(\varepsilon_{\varphi \varphi}+v_{r \varphi} \varepsilon_{r r}\right)
\end{aligned}
$$

where $E_{q}$ denotes the Young's modulus in the hoop direction, $v_{r \varphi}$ denotes the Poisson's ratio, $k$

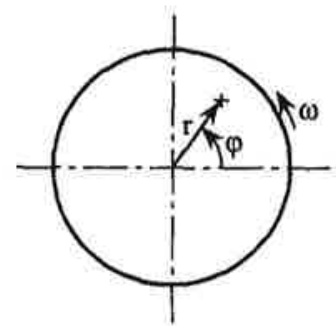

(a)
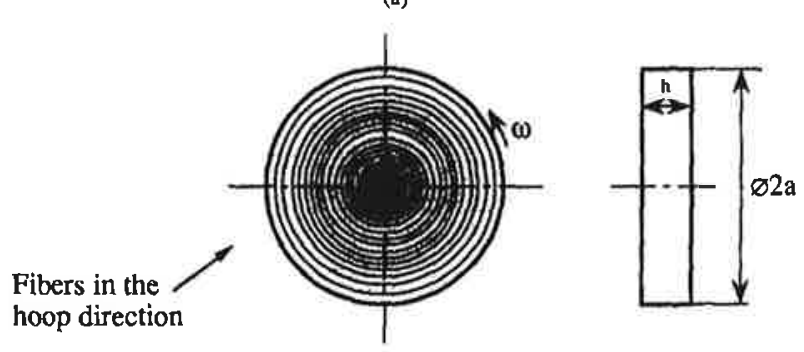

(b)
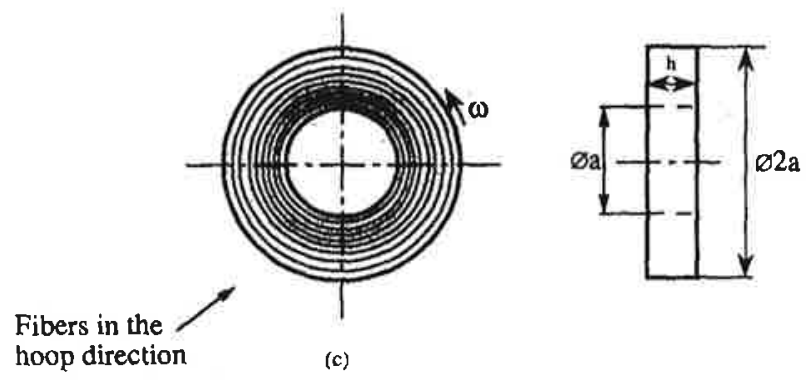

hoop direction

(c)

Fig. 2. Analyzed structures. The quantity $\omega$ represents the angular velocity: (a) coordinate system; (b) spinning disk; (c) spinning ring. denotes the ratio of the Young's modulus in the fiber direction $\left(E_{\varphi}\right)$ to the Young's modulus in the radial direction $\left(E_{r}\right)$, and $D_{\varphi}$ denotes the damage variable modeling the fiber degradation. The damage variable can be defined as the percentage of broken fibers. ${ }^{18-20}$ The percentage of broken fibers is given by the corresponding cumulative failure probability which will be modeled by a Weibull expression. ${ }^{21}$ A first expression of the damage variable can be obtained ${ }^{20}$ by extending a study of fiber-bundle to $2 \mathrm{D}$ configurations, and will be referred to as model No. 0

$$
\begin{aligned}
D_{\varphi}^{(0)}= & 1-\exp \left\{-\frac{r}{r_{0}}\left\{\frac{\sigma_{\varphi \varphi}}{\left(1-D_{\varphi}^{(0)}\right) f \sigma_{0}}\right\}^{m}\right\} \\
& \text { if } \varepsilon_{\varphi \varphi \varphi}>0 \text { and } \dot{\varepsilon}_{\varphi \varphi}>0
\end{aligned}
$$

where $m$ and $\sigma_{01}$ are the shape and scale parameters of a Weibull law, $2 \pi r_{0}$ is the corresponding gauge length, $r$ is the fiber radius (since the fiber length is $2 \pi r$ ) and $f$ is the volume fraction of fibers in the hoop direction. This first expression corresponds to a generalization of the behavior of a fiber-bundle in the hoop direction embedded in a matrix. This means that if a fiber breaks, it will not be able to carry any load (i.e. no distributed pullout takes place) and therefore this model constitutes a lower bound of the expected behavior of the composite.

On the other hand, it has been proven ${ }^{22}$ that due to distributed pull-out, the evolution of fiber breakage is not dictated by the length of the fiber (here $2 \pi r$ ) but by the length over which the tensile stress field recovers its original level. Using a shear lag approach ${ }^{23-24}$ with constant interfacial shear stress $\tau$, this length, also called recovery length, $L_{\text {rec }}$, is given by (Fig. 3)

$$
L_{\mathrm{tec}}=\frac{R T}{\tau}
$$

where $R$ is the fiber radius, $T$ is the tensile stress in the unbroken fibers. In this case, the relevant length to consider is the recovery length $L_{\text {rec }}$. By equilibrium considerations, the tensile stress $T$ is related to the external applied stress $\sigma_{\varphi \varphi}$ by

$$
f T=\frac{\sigma_{\psi \psi}}{\left(1-D_{q}\right)}
$$

Therefore, instead of $r$ in eqn (15), we will consider $L_{\text {rec }}$. We then end up with a second type of damage evolution which will be referred to as model No. 1 


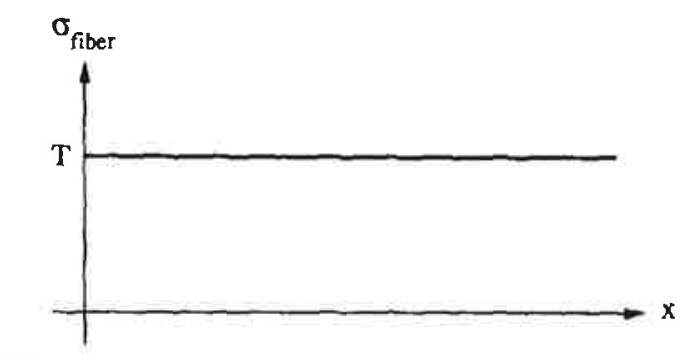

(a)

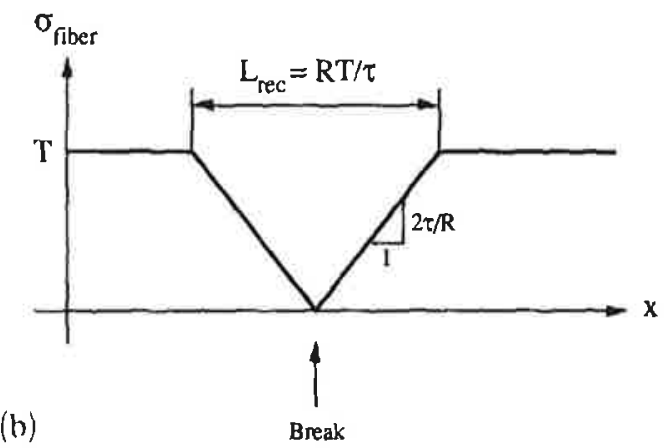

Fig. 3. Definition of the recovery length. Stress in the fiber against fiber axis $x$ : (a) just before the break; (b) just after the break.

$$
\begin{aligned}
& D_{\psi}^{(1)}= 1-\exp \left[-\left\{\frac{\sigma_{\varphi \psi}}{\left(1-D_{\varphi}^{(1)}\right) f \sigma_{\mathrm{c}}}\right\}^{m+1}\right] \\
& \text { if } \varepsilon_{\psi \psi}>0 \text { and } \dot{\varepsilon}_{\varphi \psi}>0
\end{aligned}
$$

where the expression of the scaling $\sigma_{c}$ stress is given by

$$
\sigma_{\mathrm{c}}=\left(\frac{\sigma_{0}^{m} 2 \pi r_{0} \tau}{R}\right)^{1 / m+1} .
$$

This stress has been previously used by Phoenix et al., ${ }^{25-26}$ and by Curtin ${ }^{27}$ and is referred to as characteristic strength. It can also be noted that this expression is valid under a global loadsharing hypothesis, i.e. when a fiber breaks, the load it was carrying is evenly distributed to the unbroken fibers. Although shear is involved on a microscale, we assume that the macroscopic stress state is shear-free so that eqn (5) still applies. Therefore, all of the results derived in Section 2.2 can be used.

With these two models the ultimate tensile strength in the fiber direction is given by

$$
\sigma_{\psi \psi}=\sigma_{u}=\left\{\begin{array}{l}
f \sigma_{0}\left(\frac{r_{11}}{\mathrm{e} m r}\right)^{1 / m} \text { for model No. } 0 \\
f \sigma_{c}\left(\frac{1}{\mathrm{e}(m+1)}\right)^{1 / m+1} \begin{array}{l}
\text { for model } \\
\text { No. } 1 .
\end{array}
\end{array}\right.
$$

It is worth noting that the ultimate tensile strength in the fiber direction does depend on the fiber length for model No. 0, whereas it is independent of the fiber length for model No. 1. Equation (19) shows that the characteristic strength $\sigma_{0}$ corresponds to the scaling stress of the ultimate tensile strength in the fiber direction. In particular, this result shows that the ultimate strength is independent of the total length of the fiber. Instead it depends on the recovery length at the ultimate, which is proportional to the characteristic length ${ }^{27}$ $L_{\mathrm{c}}=R \sigma_{\mathrm{c}} / \tau$. Furthermore, in this paper only the fiber breakage mechanism is taken into account since it is the most important mechanism compared to the pull-out mechanism in terms of the contribution to the stress levels. ${ }^{22-29}$ The damage level at the ultimate tensile point is given by

$$
D_{\varphi}=D_{c}=\left\{\begin{array}{l}
1-\exp \left(\frac{-1}{m}\right) \text { for model No. } 0 \\
1-\exp \left(\frac{-1}{m+1}\right) \text { for model No. } 1 .
\end{array}\right.
$$

In both models it can be noticed that the percentage of broken fibers at the ultimate tensile point $\left(D_{c}\right)$ is independent of all material parameters but the Weibull modulus.

For both models the tangent operator $\boldsymbol{H}$ takes the form

$$
\begin{aligned}
& H_{r r r r}=F_{1}-\frac{F_{2} F_{4} F_{3}^{(i)}}{1+F_{5} F_{3}^{(i)}} \\
& H_{\varphi \varphi \varphi \varphi \varphi}=\frac{F_{6}}{1+F_{5} F_{3}^{(i)}} \\
& H_{r r \varphi \varphi \varphi}=H_{\varphi \varphi r r r}=v_{r \varphi} H_{\varphi \varphi \varphi \varphi} \\
& H_{r \varphi r \varphi}=2 G_{r \varphi}>0
\end{aligned}
$$

where $G_{r q}$ corresponds to the shear modulus of the composite (assumed to be constant), the explicit expressions for $F_{j}$ are given in Appendix 1; the superscript $(i)$ refers to either model No. $0(0)$ or model No. 1 (1). It is worth noting that all conditions presupposed in the property derived in Section 2 are satisifed. Furthermore, it is easily seen that when $D_{\varphi}$ is equal to $D_{\mathrm{c}}$ then $F_{3}^{(i)}$ tends to infinity, and thus $H_{\psi \varphi \varphi \varphi \varphi}$ vanishes. On the other hand, $H_{r r r}$ and $H_{r q r 4}$ remain positive. Consequently, localization and loss of uniqueness 
occur if and only if

$$
\begin{aligned}
& D_{\varphi}=D_{\mathrm{c}} \\
& \sigma_{\varphi \varphi \varphi}=\sigma_{\mathrm{u}} .
\end{aligned}
$$

These two criteria are easier to compute than the general criteria of localization and loss of uniqueness. Moreover, the criterion refers to a maximum critical stress which does not need a computation when elasticity is coupled with damage. Indeed, an elastic computation can use criterion (22.2), whereas a computation where elasticity is coupled with damage can use both criteria. Therefore a post-processing approach described by Lemaitre $^{30}$ is not necessary, in this particular case, since criterion (22.2) does not refer to damage variables. On the other hand, as it will be shown in the next section, the damaged zone is not small compared to the dimensions of the structures. Therefore a locally coupled approach ${ }^{30}$ is not very interesting in this particular case.

It is also worth noting that criteria (22) are independent of the strain ratio $\alpha(5)$ and therefore the knowledge of the ultimate tensile strength is particularly crucial. The angle at localization is equal to $\pi / 2$, i.e. perpendicular to the fiber direction (Fig. 4(a)).

If the fibers are in the radial direction, then using the corollary of the property derived in Section 2, we find that localization and loss of

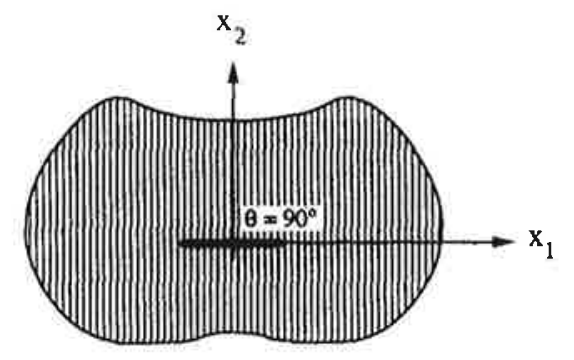

(a)

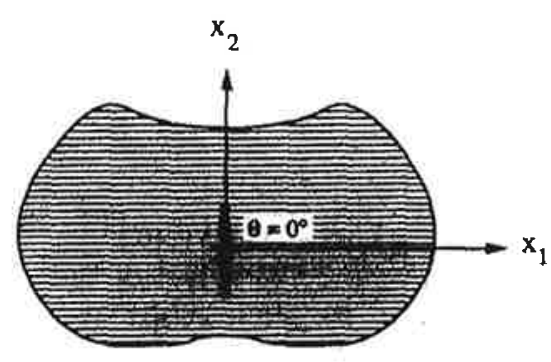

(b)

Fig. 4. Localization angles $\theta$ for: (a) fibers parallel to the 2 -axis $(\theta=\pi / 2)$; (b) fibers parallel to the 1 -axis $(\theta=0)$. uniqueness can be characterized by

$$
\begin{aligned}
& D_{1}=D_{\mathrm{c}} \\
& \sigma_{r r}=\sigma_{\mathrm{u}}
\end{aligned}
$$

where $D_{r}$ denotes the damage variable modeling the degradation of the fibers in the radial direction. The angle at localization is equal to 0 , i.e. again perpendicular to the fiber direction (Fig. $4(\mathrm{~b}))$.

\section{INITIATION OF MACROCRACKS IN SPINNING STRUCTURES}

As mentioned earlier, we will apply these results to two spinning geometries (Figs 2(b) and 2(c)). In each case, the external boundaries are supposed to be traction-free. The first part of this section is devoted to the direct application with the two models presented in Section 3. The second part deals with a simplified approach using elastic computations. The third part is concerned with the comparisons between the previous computations and the assessment of the reliability of those structures using a Weibull-type approach.

\subsection{Fully coupled computations}

When the constitutive equations presented in Section 3 are considered, it proved impossible to derive a closed-form solution for the stress state and the damage state. Instead, the problem is solved using the Finite Element Method. Constitutive laws (13)-(15), and (18) are implemented into a standard finite element code ABAQUS, ${ }^{31}$ and a solution is sought for by discretizing the problem using two-node axisymmetric shell elements. Since the linear tangent modulus $\boldsymbol{H}$ had to be implemented into the finite element code, the load (i.e. the angular rotation speed) required for loss of uniqueness and localization could be conveniently calculated using ABAQUS through a UMAT routine. In summary, the complete constitutive equations given by damage coupled with elasticity were implemented. Therefore these computations will be referred to as fully coupled.

It should be noted that due to the non-explicit expression for the damage parameter given in (15) and (18), an iterative procedure had to be outlined to determine the damage state characterized by $D_{\varphi}$ at every time the calculated strain field changed at a certain Gauss point. This was done by using a standard bisection method. A test of 
mesh dependence of the numerical results is also performed. The number of elements proves to have a very weak influence on the solution, and satisfactory results for the stress and state variable can be obtained by modeling the disk with only 20 elements.

The material analyzed herein is a CMC defined in Appendix 2. The numerical values are close to those found by Jansson and Leckie ${ }^{32}$ for a lithium alumino-silicate (LAS) matrix reinforced with silicon carbide fibers (commercial name Nicalon).

In Fig. 5, the stress field in the hoop direction obtained with a fully coupled computation is compared to the elastic stress field at the same load level. It can be noted that the load level corresponds to the localization speed for the ring with model No. 1. Due to the damage coupling, a redistribution takes place. In particular it is worth noting that the stress levels in the most loaded part $(r \geq 0.23 \mathrm{~m})$ are larger in the elastic computation. This trend is the same for both models and both structures.

In Fig. 6, the outer displacement of the ring obtained by an elastic computation and a fully coupled computation (model No. 1) are compared. Due to the gradual distribution of the damage all over the structure, the 'global' stiffness of the damaged structure softens as the applied load increases. The higher the load, the higher the softening.

In terms of load levels at localization, Table $1 a$ summarizes the results. Note that model No. 1 gives much higher load levels than model No. 0. This is mainly due to the radial dependence of the ultimate tensile strength (19) of model No. 0, compared to the radial independence of model No. 1. In Figs 7 and 8 where the stresses in the hoop

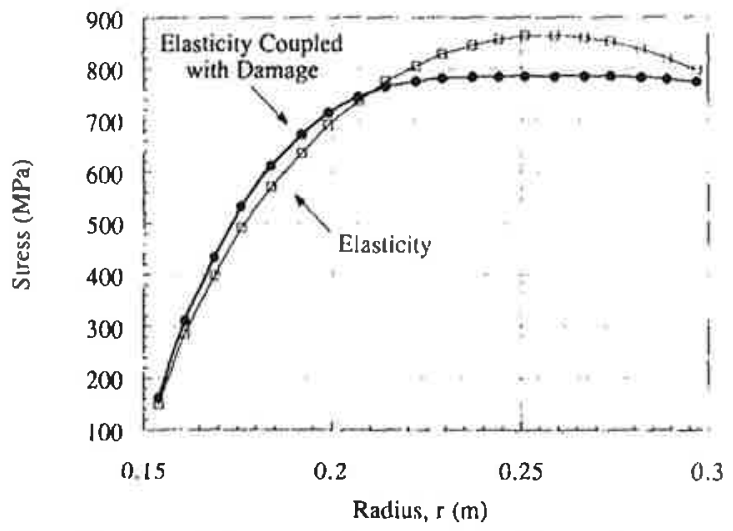

Fig. 5. Hoop stress $\sigma_{\psi y}(r)$ as a function of the radius obtained by fully coupled computation and a decoupled computation at localization $\left(\rho \omega^{2}=2 \cdot 2610^{111} \mathrm{~kg} \mathrm{~m}^{-3} \mathrm{~s}^{-2}\right)$ for the ring wih model No. 1 . Note the stress redistribution. direction are plotted against the radius, the stress levels at localization (i.e. when the curve $\sigma_{\varphi \varphi}(r)$ intersects the curve $\sigma_{\mathrm{u}}$ : criterion (22.2)) are in the same ratio as the load levels. It is worth noting that, as mentioned earlier, the ultimate tensile strength is $r$-dependent for model No. 0 (Fig. 7) and $r$-independent for model No. 1 (Fig. 8). Crite-

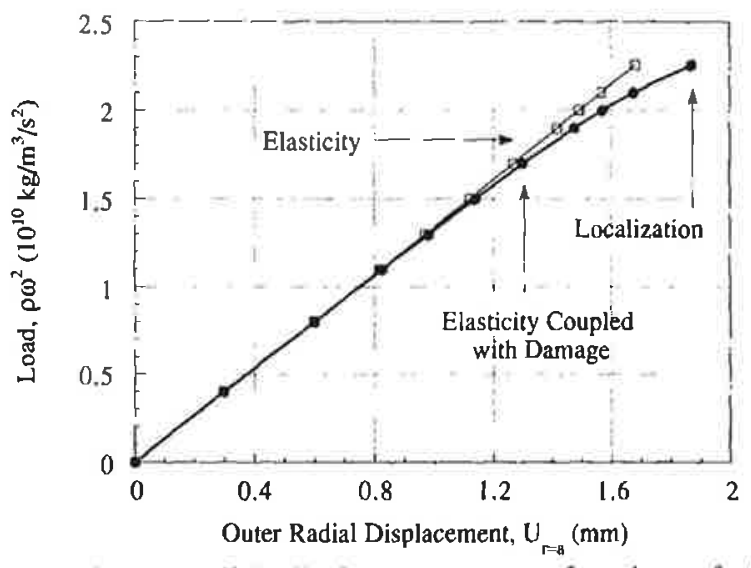

Fig. 6. Outer radial displacement as a function of the applied load $\left(\rho \omega^{2}\right)$ obtained by a fully coupled computation and a decoupled computation up to localization $\left(\rho \omega^{2}=2 \cdot 26\right.$ $\left.10^{10} \mathrm{~kg} \mathrm{~m}^{-3} \mathrm{~s}^{-2}\right)$ for the ring with model No. 1 . Note the stiffness softening.

Table 1a. Load levels $\left(\rho \omega^{2}\right)$ at localization for the two structures and the two models predicted by the fully coupled approach

\begin{tabular}{lll}
$\rho \omega^{-}$ & Disk & Ring \\
$\left(10^{10} \mathrm{~kg} \mathrm{~m}^{-3} \mathrm{~s}^{-2}\right)$ & & \\
\hline Model No. 0 & 0.59 & 0.71 \\
Model No.1 & 1.87 & 2.26 \\
\hline
\end{tabular}

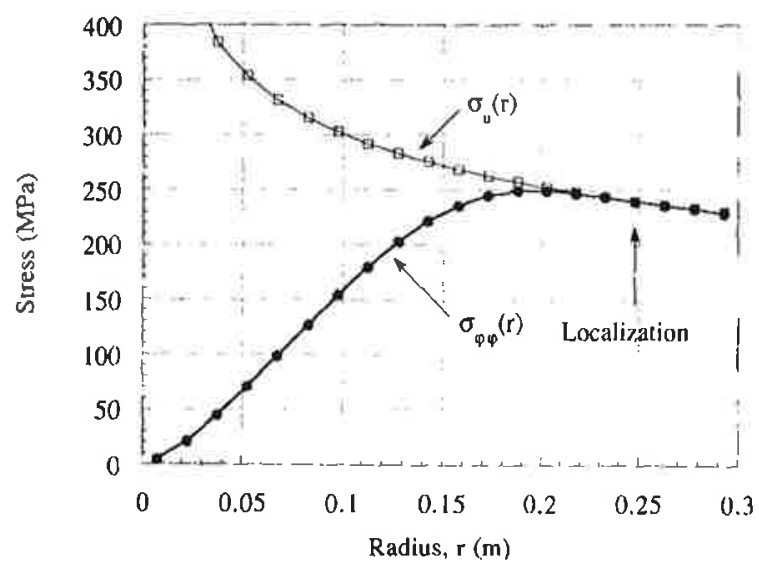

Fig. 7a. Hoop stress $\sigma_{4 r}(r)$ as a function of the radius intersecting (see the arrow) the $r$-dependent ultimate tensile strength $\sigma_{\mathrm{u}}(r)$ at localization $\left(\rho \omega^{2}=0.5910^{10} \mathrm{~kg} \mathrm{~m}^{-3} \mathrm{~s}^{-2}\right)$ for a fully coupled computation applied to the disk with model No. 0 . Note the r-dependence of the ultimate tensile strength. 


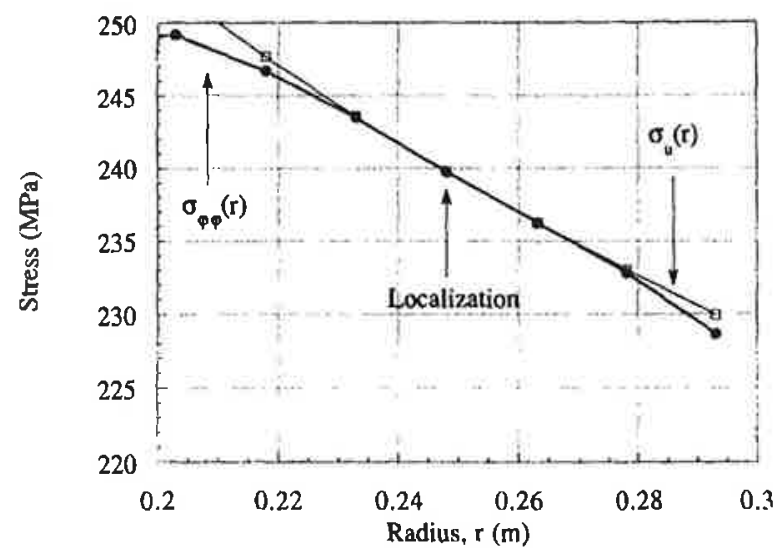

Fig. 7b. Zoom around the radius at localization of the hoop stress $\sigma_{\psi \varphi}(r)$ as a function of the radius intersecting (see the arrow) the $r$-dependent ultimate tensile strength $\sigma_{u}(r)$.

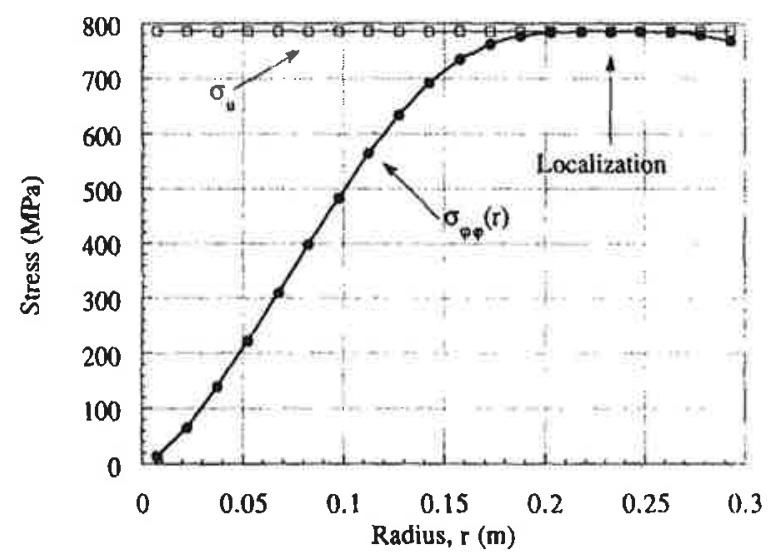

Fig. 8a. Hoop stress $\sigma_{\varphi \varphi}(r)$ as a function of the radius intersecting (see the arrow) the $r$-independent ultimate tensile strength $\sigma_{\mathrm{u}}$ at localization $\left(\rho \omega^{2}=1.87 \mathrm{~kg} \mathrm{~m}^{-3} \mathrm{~s}^{-2}\right)$ for a fully coupled computation applied to the disk with model No. 1. Note the $r$-independence of the ultimate tensile strength.

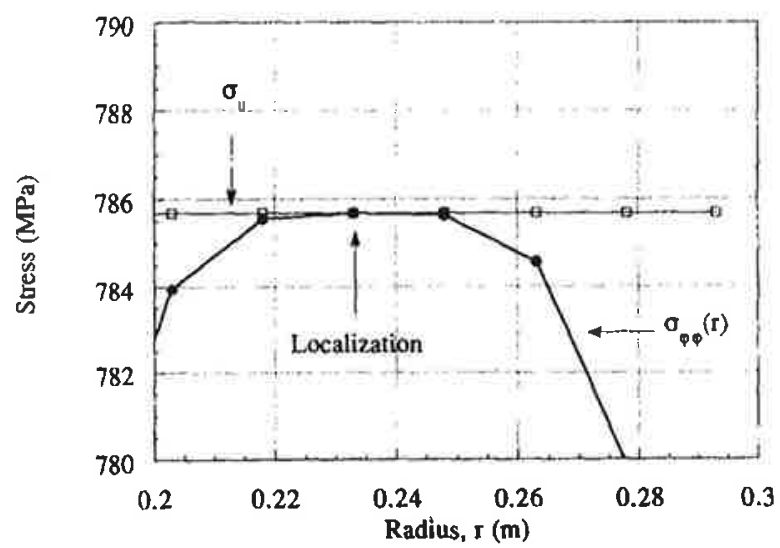

Fig. 8b. Zoom around the radius at localization of the hoop stress $\sigma_{\text {ur }}(r)$ as a function of the radius intersecting (see the arrow) the $r$-independent ultimate tensile strength $\sigma_{\mathrm{u}}$. rion (22.1) is shown in Figs 9 and 10 for model No. 0 and No. 1, respectively; note the different values for critical damage. In this case the localization point is very easy to spot compared to Figs 7 and 8.

Although the load levels in terms of $\rho \omega^{2}$ at localization are very different for model No. 0 and 1 , it can be noticed that when the dimensionless parameter $\rho \omega^{2} a^{2} / \sigma_{\mathrm{u}}\left(r_{\mathrm{loc}}\right)$ is studied (where $\sigma_{\mathrm{tu}}\left(r_{\mathrm{loc}}\right)$ denotes the ultimate tensile strength at the localization radius) the results between model No. 0 and No, 1 come close together and the main change is given by the ratio $b / a$ (Table $1 b)$. In first approximation, a good estimate for the dimensionless parameter $\rho \omega^{2} a^{2} / \sigma_{\mathrm{u}}\left(r_{\text {loc }}\right)$ is 2.1 for the disk $(b / a=0.0)$ and 2.6 for the ring $(b / a=0.5)$.

Finally, the accuracy of the numerical investigations is addressed. Figure 11 shows that the radius

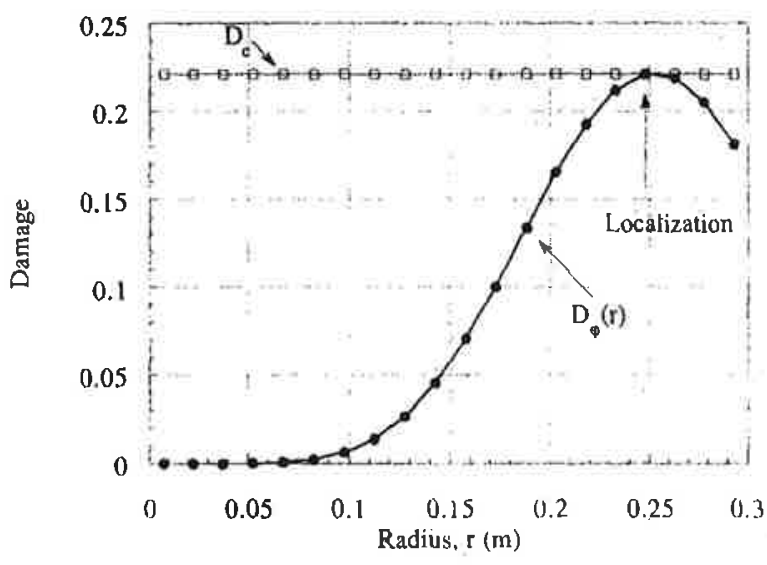

Fig. 9. Damage variable $D_{\varphi}(r)$ as a function of the radius intersecting (see the arrow) the critical damage value at localization $\left(\rho \omega^{2}=0.5910^{10} \mathrm{~kg} \mathrm{~m}^{-3} \mathrm{~s}^{-2}\right)$ for a fully coupled computation applied to the disk with modet No. 0 .

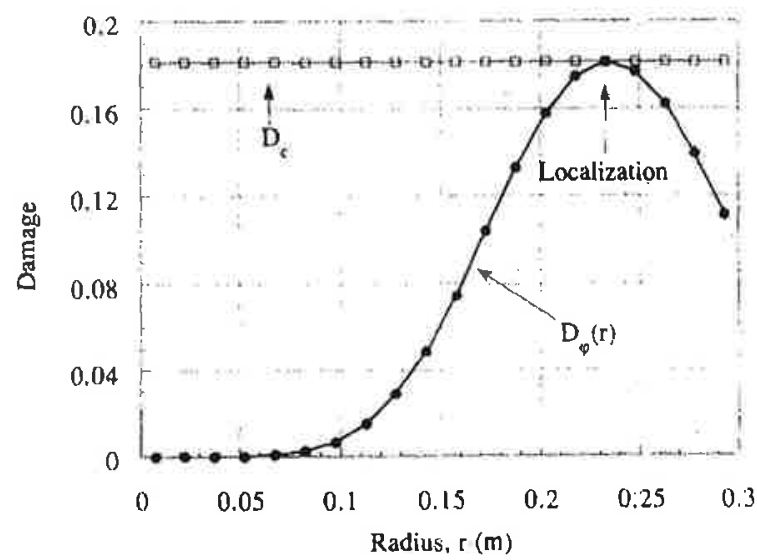

Fig. 10. Damage variable $D_{4}(r)$ as a function of the radius intersecting (see the arrow) the critical damage value at localization $\left(\rho \omega^{2}=1.8710^{16} \mathrm{~kg} \mathrm{~m}^{-3} \cdot \mathrm{s}^{12}\right)$ for a fully coupled computation applied to the disk with mode No. 1 . 
at localization is well characterized over the range of loads exceeding the localization load level. On the other hand, in terms of damage at localization (Fig. 12) and hoop stress at localization (Fig. 13), localization needs to be spotted more precisely. This phenomenon is more important in terms of angle at localization (Fig. 14). In this case, the number of decimal positions to get an angle at localization of $89^{\circ}$ (instead of $90^{\circ}$ ) is equal to 8 !

In summary, from a numerical perspective, localization needs to be spotted very accurately,

Table $1 \mathrm{~b}$. Normalized load levels $\left(\rho \omega^{2} a^{2} / \sigma_{u}\left(r_{l o c}\right)\right)$ at localization of the two structures and the two models predicted by the fully coupled approach

\begin{tabular}{lcc}
\hline$\rho \omega^{2} a^{2} / \sigma_{u}\left(r_{1 \omega 0}\right)$ & Disk & Ring \\
\hline Model No. 0 & $2 \cdot 2$ & $2 \cdot 7$ \\
Model No. 1 & $2 \cdot 1$ & $2 \cdot 6$ \\
\hline
\end{tabular}

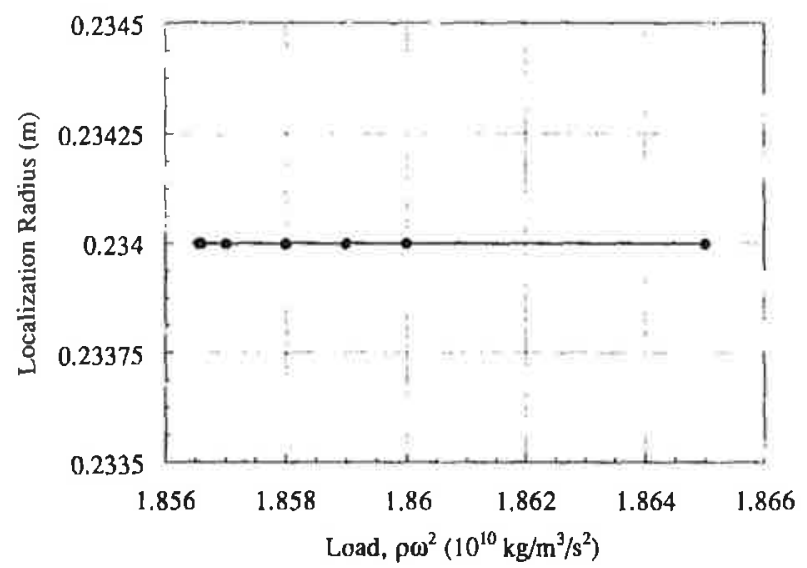

Fig. 11. Localization radius as a function of the load lcvel, for a fully coupled computation applied to the disk with model No. 1.

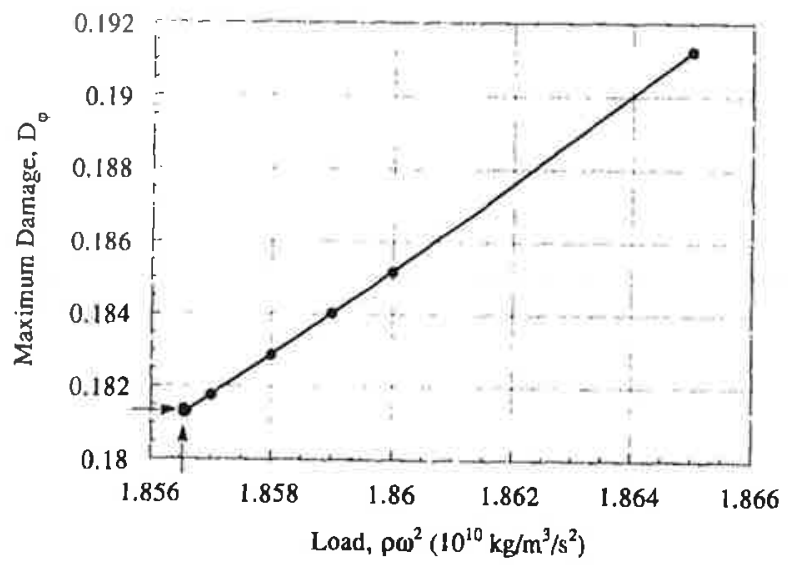

Fig. 12. Maximum damage at localization as a function of the load level, for a fully coupled computation applied to the disk with model No. 1 .

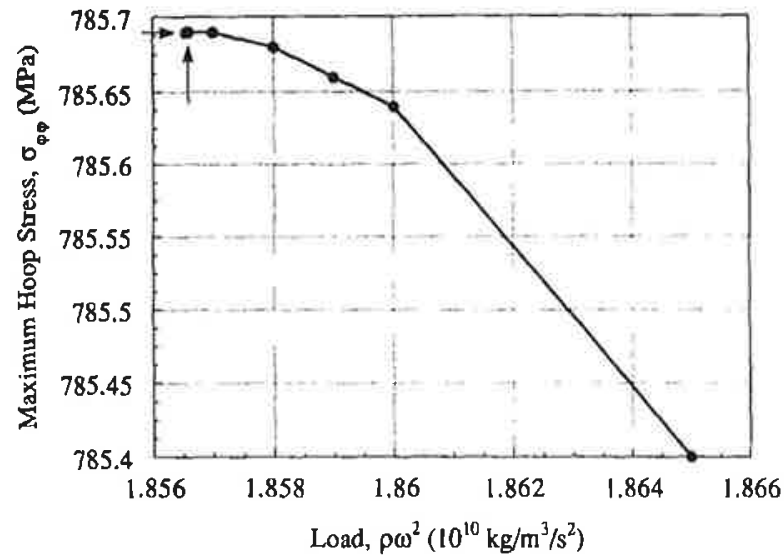

Fig. 13. Maximum hoop stress at localization as a function of the load level, for a fully coupled computation applied to the disk with model No. 1 .

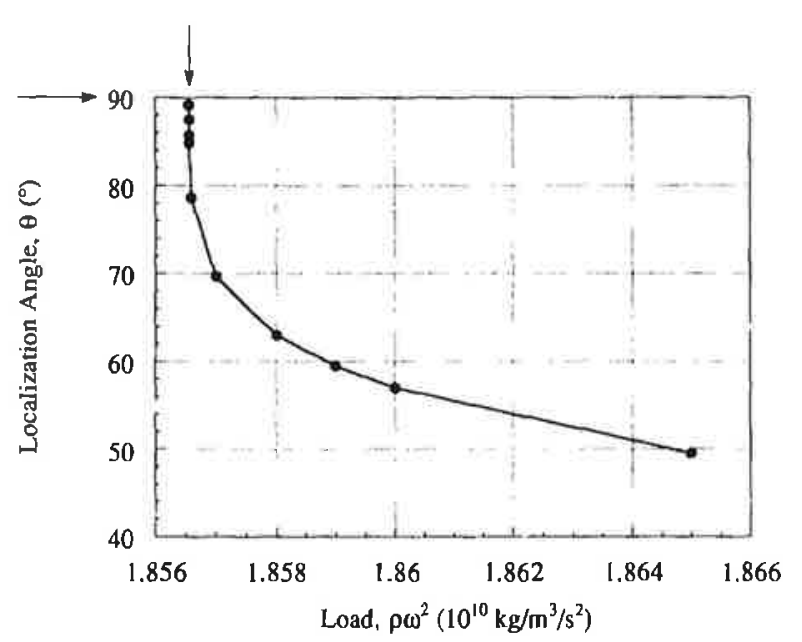

Fig. 14. Localization angle as a function of the load level, for a fully coupled computation applied to the disk with model No. 1 .

especially in terms of angle at localization. This phenomenon is only due to numerical accuracy. Statistical fluctuations do not enter this problem since the constitutive laws (on a macroscale) are deterministic, although they are based upon statistical information (on a microscale). On a microscale, however, there will be statistical fluctuations leading to a post-localization behavior, strongly dependent on these fluctuations, whereas the prelocalization behavior will not lead to fluctuations.

\subsection{Decoupled computations}

In this sub-section, we will take advantage of the criterion (22.2) which does not require a fully coupled computation. Therefore a purely elastic set of computations has been carried out. The aim of this section is to compare the load levels predicted by the two types of approach. The results 
are summarized in Table $2 a$. It can be noticed that all of the load levels at localization are lower for the decoupled analysis. This is due to redistribution, noticed earlier in Figs 7 and 8. Moreover, the error measured as the ratio of the difference between the decoupled angular speed and the fully coupled angular speed to the fully coupled angular speed is lower in the case of model No. 1. This is due to a lower stress redistribution at localization for model No. 1 since the value of critical damage is equal to $0.181(=1-\exp (-1)$ 5)) compared to model No. 0 for which the critical damage is equal to $0.221(=1-\exp (-1 / 4))$.

Again the load levels in terms of $\rho \omega^{2}$ at localization are very different for model No. 0 and No. 1 , but in terms of the dimensionless parameter $\rho \omega^{2} u^{2} / \sigma_{u}\left(r_{\mathrm{loc}}\right)$, the results are very close and the main change is given by the ratio $b / a$ (Table $2 b$ ). In first approximation, a good estimate for the dimensionless parameter $\rho \omega^{2} a^{2} / \sigma_{\mathrm{u}}\left(r_{\mathrm{loc}}\right)$ is $2 \cdot 0$ $(b / a=0.0)$ for the disk and 2.5 for the ring $(b /$ $a=(0 \cdot 5)$. These results constitute a lower bound when compared to the fully coupled results.

The maximum difference between the two models approaches is $-6.1 \%$, which is not significant. Therefore a decoupled computation gives good information of the loads that these kind of structures can support. Furthermore, due to the stress field redistribution, the decoupled approach gives conservative results of all of the analyzed cases. Therefore it constitutes a 'good' lower bound approximation of the models No. () and No. 1.

Table 2a. Load levels $\left(\rho \omega^{2}\right)$ at localization for the two structures and the two models predicted by the decoupled approach, in parenthesis () is the difference in terms of angular velocity with the fully coupled approach

\begin{tabular}{lcc}
\hline & \multicolumn{2}{c}{$\rho \omega^{2}\left(10^{11} \mathrm{~kg} \mathrm{~m}^{-3} \mathrm{~s}^{-1}\right)$} \\
\cline { 2 - 3 } & Disk & \multicolumn{1}{c}{ Ring } \\
\hline Model No. 0 & $0.53(-4.6 \%)$ & $0.62(-6.1 \%)$ \\
Model No. 1 & $1.75(-3.1 \%)$ & $2 \cdot(0.5(-4.5 \%)$ \\
\hline
\end{tabular}

Table $2 b$. Normalized load levels $\left(\rho \omega^{2} a^{2} / \sigma_{u}\left(r_{l o c}\right)\right)$ at localization for the two structures and the two models predicted by the decoupled approach

\begin{tabular}{lcc}
\hline & \multicolumn{2}{c}{$\rho \omega^{2} a^{2} / \sigma_{\mathrm{u}}\left(r_{\text {los }}\right)$} \\
\cline { 2 - 3 } & Disk & Ring \\
\hline Model No. 0 & $2 \cdot 1$ & $2 \cdot 5$ \\
Model No. 1 & $2 \cdot 1$ & $2 \cdot 5$ \\
\hline
\end{tabular}

\subsection{Comparison with a Weibull-type of analysis}

In this sub-section we compare the load levels at localization, obtained by both approaches, to the cumulative failure probability obtained by modeling the material as an elastic brittle material. The conditions for first failure are determined at the load levels obtained previously. We assume that the disk and the ring are made of a monolithic ceramic whose characteristics are identical to that of the fibers (i.e. same $m, \sigma_{0}$, and $r_{0}$ ). This kind of analysis may be used to assess the reliability of these spinning structures, supposedly made of brittle material. In the framework of the weakest link assumption, the expression of the cumulative failure probability $P_{F}$ is given by a Weibull expression $^{21}$

$$
P_{\mathrm{r}}=1-\exp \left\{-\frac{h}{V_{0}} \int_{S}\left\{\frac{\sigma_{\varphi \psi}}{\sigma_{01}}\right\}^{\prime \prime \prime} \mathrm{d} A\right\}
$$

where $V_{0}$ is the gauge volume $\left(V_{0}=h \pi r_{i j}^{2}\right)$, and $h$ the disk or ring thickness. Using the same normalization as in the previous sections, the elastic stress field can be scaled by writing the hoop stress as follows

$$
\sigma_{\psi r}(r)=\left(\rho \omega^{2} a^{2}\right) \mathrm{f}(r)
$$

where the function $\mathrm{f}$ is a dimensionless function. Since the function $f$ depends only on the shape of the stress field and not on its level, it can characterize the stress field heterogeneity; and the cumulative failure probability can be rewritten as

$$
P_{\mathrm{l}}=1-\exp \left\{-\frac{A}{A_{\mathrm{ll}}} H_{\mathrm{m}}\left(\frac{\rho \omega^{2} a^{2}}{\sigma_{0}}\right)^{m}\right\}
$$

where $H_{\mathrm{m}}$ is a generalized definition of the stress heterogeneity factor ${ }^{33}$ associated with a Weibull law, and is defined as

$$
H_{\mathrm{m}}=\frac{1}{a^{2}-b^{2}} \int_{b}^{\prime \prime} 2 r\{\mathrm{f}(r)\}^{\prime \prime \prime} \mathrm{d} r .
$$

Note that if the hoop stress is homogeneous then $H_{\mathrm{m}}$ is equal to 1; otherwise, as the stress field gets more heterogeneous, $H_{\mathrm{m}}$ gets closer to zero. In the case of the disk $H_{\mathrm{m}}=0.04$ and in the case of the ring $H_{m}=0.02$. The stress heterogeneity factors fully characterize the elastic stress field in terms of cumulative failure probability, i.e. in terms of reliability of these structures.

The results of cumulative failure probability for the same load levels as those predicted by the fully 
coupled approach are summarized in Table 3. It can be noticed that the cumulative failure probabilities are very high. Therefore a design strategy using this kind of reliability assessment can expect quite high value in terms of the cumulative failure probability corresponding to the load levels at localization.

\section{CONCLUSIONS}

In the framework of Continuum Damage Mechanics, a localization criterion corresponds to the initiation of a macrocrack. Under certain hypotheses, the localization criterion can be rewritten in terms of a necessary and sufficient condition in terms of one component of the tangent operator. This result is applied to derive criteria at localization in terms of a critical damage value and a maximum normal stress for constitutive laws modeling the fiber breakage for unidirectionally reinforced ceramic matrix composites.

The constitutive laws are used to study axisymmetric spinning structures made of CMCs. Two approaches are analyzed. The fully coupled approach where the complete constitutive law is implemented in a FE code is compared to the decoupled approach, that consists of using an elastic computation. Indeed, one of the previous criteria can be used in an elastic computation. The result predicting localization are of the same order of magnitude. Furthermore, the decoupled approach gives conservative results in terms of load at localization, and the results in the analyzed structures are very close to those predicted by a fully coupled method. The fact that the decoupled approach gives conservative results is due to a stress redistribution when the fully coupled approach is used.

This kind of approach will also be carried out in the case of structures reinforced by fibers in two perpendicular directions. This enables us to

Table 3. Cumulative failure probability corresponding to the localization load levels obtained by the decoupled and fully coupled approaches

\begin{tabular}{lcc}
\hline & \multicolumn{2}{c}{$P_{\mathrm{F}}$} \\
\cline { 2 - 3 } & Disk & Ring \\
\hline Model No. & 0.60 & 0.53 \\
Model No. 1 & 1.00 & $1 \cdot 00$ \\
\hline
\end{tabular}

look for an optimum volume fraction of fibers in terms of load at localization.

\section{ACKNOWLEDGEMENTS}

The authors gratefully acknowledge the financial support of the US Air Force, through contract AFOSR-90-0132 with the Department of Mechanical and Environmental Engineering, University of California at Santa Barbara, and the support by the Advanced Research Project Agency, through the University Research Initiative.

\section{REFERENCES}

1. U.R.I., Proceedings University Research Initiative, University of California, Santa Barbara (1992).

2. Aveston, J., Cooper, G. A. \& Kelly, A., Single and multiple fracture. In Conf. Proceedings of the National Physical Laboratory: Properties of Fiber Composites. IPC Science and Technology Press, Surrey, UK (1971).

3. Budiansky, B., Hutchinson, J. W. \& Evans, A. G., Matrix tracture in fiber-reinforced ceramics. J. Mech. Phys. Solids, 34 (1986) 167-89.

4. Budiansky, B., Tensile strength of aligned-fiber composites. Proceedings University Research Initiative, Winter Study Group. University of California. Santa Barbara (1993).

5. Coleman, B. D., On the strength of classical fibers and fiber bundles. J. Mech. Phys. Solids, 7 (1958) 60-70.

6. Lemaitre, J., A Course on Damage Mechanics, SpringerVerlag, Berlin (1992).

7. Billardon, R. \& Doghri, I., Prévision de l'amorçage d'une macro-fissure par la localisation de l'endommagement. C. R. Acad. Sci. Paris, 308 (II) (1989) 347-52.

8. Benallal, A., Billardon, R. \& Geymonat, G., Localization phenomena at the boundaries and interfaces of solids. Proc. 3rd Conf. on Constitutive Laws for Engineering Materials: Theory and Applications, Tucson, AZ (1991).

9. Hadamard, J., Leçon sur la propagation des ondes et les équations de l'hydrodynamique, Paris (1903).

10. Hill, R., Acceleration waves in solids. J. Mech. I'hys. Solids, 10 (1962) 1-16.

11. Mandel, J., Ondes plastiques dans un millicu indéfini à trois dimensions. J. de Mécanique. 1 (1) (1962) 3-30.

12. Rice, J. R., The localization of plastic deformations. Proc. Theoretical and Applied Mechanics, Koiter, W. T. (1976).

13. Borré, G. \& Maier, G., On linear versus nonlinear flaw rules in strain localization analysis. Meccanica, 24 (1989) 36-41.

14. Rudnicki, J. W. \& Rice, J. R., Conditions for localization of deformation in pressure-sensitive dilatant materials. $J$. Mech. Phys. Solids, 23 (1975) 371-94.

15. Rice, J. R. \& Rudnicki, J. W., A note on some features of the theory of localization of deformation. Int. J. Solids Struct, $16(1980)$ 597-605.

16. Ortiz, M., Leroy, Y. \& Needelman, A., A finite element method for localized failure analysis. Comput. Meths Appl. Engng., 61 (1987) 189-214.

17. Hild, F., Larsson, P.-L. \& Leckie, F. A., Localization due to damage in fiber reinforced composites. Int. J. Solids Struct., 29 (24) (1992) 3221-38. 
18. Krajcinovic, D. \& Silva, M. A. G., Statistical aspects of the continuous damage theory. Int. J. Solids Struct., 18 (7) (1982) 551-62.

19. Hult, J. \& Travnicek, L., Carrying capacity of fiber buridles with varying strength and stiffness. J. de Mécanique Théorique et Appliquée, 2 (2) (1983) 643-57.

20. Hild, F., Larsson, P.-L. \& Leckie, F. A., Localization due to damage in two-direction fiber-reinforced composites. J. Appl. Mech. (1992) accepted.

21. Weibull, W., A statistical theory of the strength of materials. Ing. Vetenskap. A kad.; 15 (1939).

22. Curtin, W. A., Theory of mechanical properties of ceramic matrix composites. J. Am. Ceram. Soc., 74 (11) (1991) 2837-45.

23. Cox, H. L., The elasticity and the strength of paper and other fibrous materials. Br. J. Appl. Phys., 3 (1952) 72-9.

24. Kelly, A., In Strong Solids. Oxford University Press, 2nd edn, Oxford University Press (1973).

25. Henstenburg, R. B. \& Phoenix, S. L., Interfacial shear strength using single-filament-composite test. Part II: A probability model and Monte Carlo simulations. Polym. Comp., 10 (5) (1989) 389-406.
26. Phoenix, S. L. \& Raj, R, Scalings in fracture probabilities for a Brittle matrix fiber composite. Acta Metall. Mater., 40 (11) (1992) 2813-28.

27. Curtin, W. A., Exact theory of fiber fragmentation in single-filament composite. J. Mat. Sci., 26 (1991) 5239-53.

28. Hild, F., Domergue, J.-M., Evans, A. G. \& Leckie, F. A., Tensile and flexural ultimate strength of fiber reinforced ceramic matrix composites. Int. J. Solids Struct., 31 (7) (1994) 1035-45

29. Phoenix, S. L., Statistical issues in the fracture of brittle matrix fibrous composites. Comp. Sci. Tech., $\mathbf{4 8}$ (1993) $65-80$.

30. Lemaitre, J., Micro-mechanics of crack initiation. Int. J. Fract., 42 (1990) 87-99.

31. Hibbitt, H. D., Karlsson, B. I. \& Sorenson, P., ABAQUS, version 5.2 (1992).

32. Jansson, S. \& Leckie, F. A., The mechanics of failure of silicon carbide fiber-reinforced glass-matrix composites. Acta Metall., 40 (11) (1993) 2967-78.

33. Hild, F., Billardon, R. \& Marquis, D., Stress heterogeneity influence on failure of brittle materials. C. $R$. Acad. Sci. Paris, 315 (II) (1992) 1293-8.

\section{APPENDIX 1}

$\begin{array}{lll}\text { Model No. } 0 & \text { No. } 1\end{array}$

$$
\begin{aligned}
& F_{1}=\frac{E_{\psi}}{k\left(1-v_{r q}^{2}\left(1-D_{q}\right) k\right)} \\
& F_{2}=\frac{E_{\varphi} \nu_{r \varphi}\left(\nu_{r \varphi} \varepsilon_{r r}+\varepsilon_{\varphi \varphi \varphi}\right)}{\left(1-v_{r \varphi}^{2}\left(1-D_{\varphi}\right) k\right)^{2}} \\
& F_{+}=\frac{E_{\psi} \nu_{r \varphi}\left(1-D_{\psi}\right)}{1-v_{r \psi}^{2}\left(1-D_{\psi}\right) k} \\
& F_{5}=\frac{E_{\psi}\left(\nu_{r \psi} \varepsilon_{r r}+\varepsilon_{\psi \psi}\right)}{\left(1-v_{r \psi}^{2}\left(1-D_{\psi}\right) k\right)^{2}} \\
& F_{6}=\frac{E_{\psi}\left(1-D_{\psi}\right)}{1-v_{r \varphi}^{2}\left(1-D_{\varphi}\right) k}
\end{aligned}
$$

$$
F_{3}^{(0)}=\frac{\frac{m}{f \sigma_{0}}\left\{\frac{\sigma_{\varphi \varphi}}{\left(1-D_{\varphi}^{(0)}\right) f \sigma_{0}}\right\}^{m-1}}{1-m\left\{\frac{\sigma_{\varphi \varphi}}{\left(1-D_{\varphi}^{(0)}\right) f \sigma_{0}}\right\}^{m}}
$$


APPENDIX 2

Material parameters for the ceramic matrix fiber composite analyzed in the finite element calculations are:

$$
\begin{aligned}
E_{r} & =20.0 \mathrm{GPa} \\
E_{\varphi} & =140.0 \mathrm{GPa} \\
G_{r \varphi} & =13.0 \mathrm{GPa}
\end{aligned}
$$

$$
\begin{aligned}
v_{r \varphi} & =0.0214 \\
m & =4.0 \\
f \sigma_{0} & =1450 \cdot 0 \mathrm{MPa} \\
r_{0} & =0.002 \mathrm{~m} \\
f \sigma_{c} & =1300 \cdot 0 \mathrm{MPa} \\
a & =0.3 \mathrm{~m}, b=0.15 \mathrm{~m} .
\end{aligned}
$$

